\title{
Quasars Do Not Live in Merging Systems: No Enhanced Merger Rate at $z \lesssim 0.8$
}

\section{Mauricio Cisternas ${ }^{1}$, Knud Jahnke ${ }^{1}$, Katherine J. Inskip ${ }^{1}$, and the COSMOS Collaboration}

\author{
${ }^{1}$ MPIA, Königstuhl 17, D-69126 Heidelberg, Germany \\ Email: cisternas@mpia.de
}

Keywords. galaxies: active, galaxies: interactions, quasars: general

We tackle the long-standing question "what is the relevance of major mergers and interactions as triggering mechanisms for AGN activity?" We study a sample of 83 quasars over $z \sim 0.3-1.0$ with high-resolution $H S T /$ ACS imaging in the F814W (broad I) band in the COSMOS field (Scoville et al. 2007), detected by their X-ray emission in the XMM-Newton survey (Hasinger et al. 2007). We perform a visual analysis of their morphologies, looking for signatures of merging and interactions in their host galaxies that could potentially be related to the AGN fueling mechanism. To get the best possible picture of the host galaxies, we remove the bright quasar by modeling each of them as a Sersic surface brightness profile plus a central point source, through a $2-\mathrm{D}$ parametric fit.

To establish the significance of the distortions seen in our AGN hosts, we compare them with a control sample of inactive galaxies, matched in redshift and brightness, and from the same dataset. We perform a blind comparison between the quasar hosts and the inactive galaxies by mocking the latter up as AGN, by adding a star as a fake active nucleus and then performing the same subtraction procedure as with the original quasars.

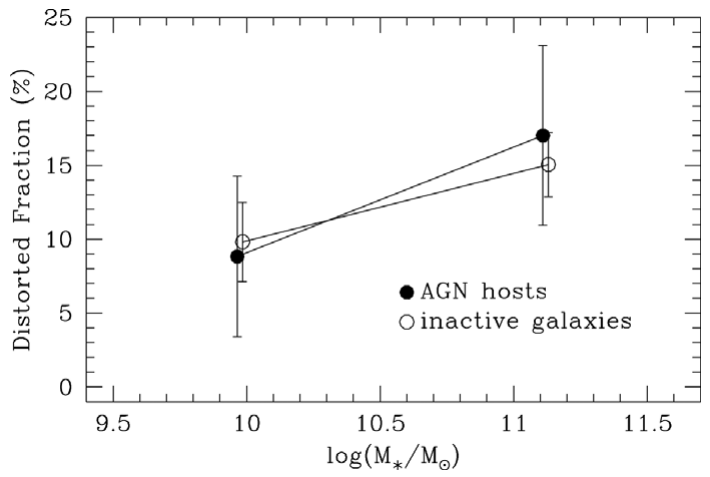

Figure 1. Fraction of heavily distorted galaxies as a function of estimated stellar mass (observed $I$-band magnitude plus assumed intermediate age stellar population). Again, we find no evidence for an excess in the distortion fractions of the AGN hosts even at the massive end.

Over all masses, we find the fractions of heavily distorted AGN host galaxies and inactive galaxies to be $14.4 \pm 8.7 \%$ and $13.9 \pm 8.0 \%$ repectively. Our results show no evidence of an enhancement in the frequency of merging signatures for AGN hosts. Even for the massive end of our sample, in which mergers could potentially play a larger role, we find no difference, as shown in Figure 1. Our results are direct evidence for the scenario suggested by Hasinger (2008). Here, the major merger-driven evolution dominates in the early universe, producing the bulk of the brightest quasars at $z=2-3$. Later, around $z \sim 1$, secular evolution and minor interactions take the lead, becoming the main fueling mechanisms.

\section{References}

Hasinger, G. 2008, A\& $A$, 490, 905

Hasinger, G., et al. 2007, ApJS, 172, 29

Scoville, N., et al. 2007, ApJS, 172, 38 